\title{
New approaches to neural circuits in behavior
}

\author{
Aleena Garner and Mark Mayford ${ }^{1}$ \\ Department of Cell Biology \& Dorris Neuroscience Center, The Scripps Research Institute, La Jolla, California 92037, USA
}

\begin{abstract}
A fundamental goal of neuroscience is to understand how the brain represents the world. If we consider the neurons of the brain to be one system and the external world to be another system, how do the two systems interact, and by what translational code does the former represent the latter? Recent advances in imaging neural activity, genetically altering specific neural circuits, and genetic tools for the direct manipulation of neural activity are beginning to shed light on this critical question. We review recent advances in these areas that illustrate a path to addressing this fundamental question.
\end{abstract}

\section{Imaging}

Recording the electrical activity of neurons in the intact brain has a long history in neuroscience and has provided an outline of the properties of neurons in different brain regions and some of the transformations in processing information between regions. While current technology allows the activity of hundreds of cells to be simultaneously recorded with high temporal resolution (Buzsaki 2004), the specific cell types giving rise to the electrical signals and the spatial relationships between them cannot be readily determined using this technique. $\mathrm{Ca}^{2+}$ imaging can reveal neural activity, albeit with somewhat reduced sensitivity and temporal resolution relative to electrophysiological techniques, but with precise spatial identification of the active neurons. This approach can be used to dissect the fine anatomical structure of neural circuits recruited to a specific behavior. Komiyama et al. (2010) used $\mathrm{Ca}^{2+}$ imaging to monitor neural activity patterns in motor cortex during sensory-motor learning. The investigators trained head-fixed mice to lick in response to one odor and to suppress licking in response to a second odor. They found task-related (e.g., lick, no lick) neural activity in the anteriorlateral motor (ALM) and posterior-medial motor (PMM) areas with no apparent anatomical clustering of the different response types. They also observed correlated activity between neurons of the same response type that decreased with the distance between the neurons. This correlated activity was observed even during inter-trial intervals, suggesting that neurons of a similar response type were locally interconnected over a limited distance. This study provides one example of how $\mathrm{Ca}^{2+}$ imaging can provide a more precise view of the circuit structure underlying behavior. In this case, it appears that neurons showing a specific response type develop increased local connectivity but that the different response types are interspersed with no apparent anatomical segregation.

$\mathrm{Ca}^{2+}$ imaging has typically used indicator dyes that can be applied both in vivo and in vitro; however, a major drawback of this approach is that it is difficult to repeatedly load cells with the indicator for prolonged studies. A key advance in this area was the development of effective genetically encoded fluorescent indicators of $\mathrm{Ca}^{2+}$ signals. Tian et al. (2009) developed a modification of GFP in which fluorescence was sensitive to $\mathrm{Ca}^{2+}$ levels and showed that it could be used effectively in awake behaving mice. This genetically encoded $\mathrm{Ca}^{2+}$ indicator (GCaMP3) can be targeted to specific cell types to allow prolonged and repeated imaging of $\mathrm{Ca}^{2+}$ signals.

\footnotetext{
${ }^{1}$ Corresponding author

E-mail mmayford@scripps.edu

Article is online at http://www.learnmem.org/cgi/doi/10.1101/lm.025049.111.
}

The hippocampus is critical for spatial learning and neurons in this structure fire in response to an animal's location in a particular environment. These place cells are thought to encode a spatial representation of the animal's surroundings. Is this spatial map of the environment reflected in an anatomically segregated map in the hippocampal place cells? To address this question, Dombeck et al. (2010) used GCaMP3 to image $\mathrm{Ca}^{2+}$ transients in hippocampal CA1 neurons of mice as they navigated through a virtual environment. The CA1 neurons were virally transfected to express the GCaMP3 protein, and the animals were head-fixed to a 2-photon microscope and allowed to run on a spherical treadmill on a virtual linear track. Under these conditions, hippocampal place cells could be identified using $\mathrm{Ca}^{2+}$ imaging with properties similar to that observed with electrophysiological recordings. Using the spatial resolution afforded by $\mathrm{Ca}^{2+}$ imaging, the investigators found that no two- or three-dimensional relationship existed between the location of a place field in the environment and the position of the corresponding place cell in CA1. In addition, no relationship was found between the correlation in activity and distance between cells. The researchers also showed the existence of well-defined place fields in apical dendrites of CA1 cells, likely due to back-propagating action potentials. Thus, similar to the motor cortex, the hippocampal representation of space has no discernible anatomical segregation.

A drawback of imaging is the requirement to head-fix the animals to a microscope objective limiting the repertoire of accessible behaviors. To allow imaging in awake behaving mice, Flusberg et al. (2008) developed a miniaturized endoscopic microscope that is small and light enough to mount on a freely moving mouse's head. They used this approach to measure dendritic $\mathrm{Ca}^{2+}$ spiking in cerebellar Purkinji cells. The average $\mathrm{Ca}^{2+}$ waveform was similar for anesthetized, awake but motionless, and actively moving mice, revealing the precision and stability of this method in behaving animals. Additionally, the investigators found increases in correlated $\mathrm{Ca}^{2+}$ spiking during movement compared with rest. Finally, they showed coherent $\mathrm{Ca}^{2+}$ activity occurring more frequently between neighboring dendritic trees than distally separated ones.

These results show examples of how recent technologies have greatly expanded the ability to investigate the spatial-temporal relationships between cellular correlates of cognition and behavior. The development of genetically encoded $\mathrm{Ca}^{2+}$ indicators for prolonged imaging combined with endoscopic microscopy to allow access to deep structures in a freely behaving animal should provide a powerful new approach for assessing circuit activity with behavior and the anatomical relationship of neurons within the circuit. While imaging is useful for assessing the activity of circuits in behavior and suggests potential sites of plasticity and computational transformations, it does not allow direct tests 
of circuit function. The recent development of a host of genetically encoded modulators of neural activity fills this gap.

\section{Manipulation of anatomically defined circuits}

The development of genetically encoded regulators of neural activity within the past 10 years has opened up the possibility of manipulating specific cell types to directly test their role in behavior. Some of these new tools are listed in Table 1. They fall into two broad categories, light-regulated and ligand-regulated receptors or channels. Activation of the channels can be used to either artificially fire or suppress the natural firing of the neurons in which they are expressed. The development and mechanism of action of these new tools have been reviewed previously (Luo et al. 2008). One of the primary distinctions is that the light-regulated approach (optogenetics) allows for precise temporal control over neural activity, while the ligand-based approach allows for control of broader anatomical regions by systemic delivery of the ligand. The ability to photonically or chemically inhibit and activate genetically defined cells will allow an investigation of necessity, sufficiency, and functional causality of the neural circuits giving rise to perception and cognition. In this section, we discuss several recent studies in which these approaches are used to alter defined brain regions through anatomically targeted delivery with viral vectors.

One of the first uses of this approach provided an elegant determination of the number of neurons that are sufficient for perception (Huber et al. 2008). Viral transfection was used to express channelrhodopsin2 (ChR2) in a random subset of pyramidal neurons in layer $2 / 3$ of the mouse barrel cortex. The activity of these cells could be controlled with single action potential resolution, and the number of neurons firing could be varied by changing the light intensity. The mice were taught to nose-poke in one of two ports for a water reward depending on whether they perceived the firing of neurons in the somatosensory cortex. When five action potentials were induced, activation of as few as 60 neurons could be detected and used in the decision-making task. When only a single action potential per cell was produced, mice could detect and respond to activity in approximately 300 neurons. Thus, the number of cortical neurons that are capable of producing a perception and driving behavior is quite small. Such a result could not have been obtained with conventional electrophysiological techniques because stimulating electrodes activate excitatory, inhibitory, and glial cells as well as local and longrange fibers of passage.

Mating and aggression are two innate behaviors critical to survival that have been linked to circuits in the hypothalamus.

Table 1. Genetically encoded regulators of neural activity

\begin{tabular}{|c|c|c|c|}
\hline Gene & Activation & Action & References \\
\hline ChR2 & Light (473 nm) & Depolarization & $\begin{array}{l}\text { Boyden et al. } \\
2005\end{array}$ \\
\hline Arch & Light (575 nm) & Hyperpolarization & Chow et al. 2010 \\
\hline eNpHR3.0 & Light (594 nm) & Hyperpolarization & Tye et al. 2011 \\
\hline AlstR & $\begin{array}{l}\text { Peptide } \\
\text { (allatostatin) }\end{array}$ & Hyperpolarization & $\begin{array}{l}\text { Lechner et al. } \\
2002\end{array}$ \\
\hline hM3Dq & Chemical (CNO) & Depolarization & $\begin{array}{l}\text { Alexander et al. } \\
2009\end{array}$ \\
\hline hM4D & Chemical (CNO) & Hyperpolarization & $\begin{array}{l}\text { Armbruster et al. } \\
2007\end{array}$ \\
\hline GluCl & Chemical (IVM) & Hyperpolarization & $\begin{array}{l}\text { Lerchner et al. } \\
\quad 2007\end{array}$ \\
\hline
\end{tabular}

Partial list of commonly used neural activity regulators. A more comprehensive list of genetically encoded regulators and their function can be found elsewhere (Luo et al. 2008). (CNO) Clozapine-N-oxide; (IVM) ivermectin.
Lin et al. (2011) used a combination of optogenetic, chemical genetic, and recording approaches to investigate the neural networks underlying these behaviors. Electrophysiological recording and $c$ fos labeling defined the ventrolateral subregion of the ventromedial hypothalamic nucleus ( $\mathrm{VMHvl}$ ) as a locus for both aggression and mating responsive units. The responsive cells were sparse and intermixed but a given unit seemed to respond in only one of the two behavioral situations. To functionally test the role of these neurons in the behavior, the investigators used CRE-dependent expression to target channelrhodopsin (ChR2) specifically into VMHvl neurons. Light-based stimulation via ChR2 of VMHvl neurons yielded different results depending on the environmental setting. Stimulation did not alter behavior when administered to an isolated mouse, but produced rapid, coordinated, and directed attack behavior when a male, female, or castrated male intruder was presented. When an anaesthetized mouse was present, illumination resulted in attack by $60 \%$ of mice unless the anaesthetized mouse was artificially moved, which resulted in attack by $100 \%$ of mice in which the VMHv1 was stimulated. In fact, illumination resulted in attack of a motionless glove by $25 \%$ of mice, but if the glove was moved, $75 \%$ of mice attacked. When a female was present, illumination yielded attack $80 \%$ of the time before initiation of mating; however, during intromission the same light intensity was ineffective. However, after ejaculation, illumination resulted in attack with the same probability as it did before mating.

These results demonstrate that activation of VMHv1 neurons is sufficient to induce aggressive behavior. To determine whether these neurons are necessary for aggression, Slimko et al. (2002) selectively silenced VMHvl neurons using genetically directed expression of the ivermectin (IVM)-gated chloride channel (GluCl), which induces hyperpolarization upon IVM binding. Silencing the VMHv1 neurons prevented offensive attack behavior but had no impact on mating behavior. These results show that synthetically evoked excitation and inhibition can drive or suppress certain behaviors depending on specific environmental circumstances. The aggressive behaviors seemed to dominate, although the behavioral responses were sensitive to the mating status of the mouse, possibly through modulation by the mating-responsive neurons in the same area.

Dopamine signaling is known to mediate a reward component of motivated behavior and is a target for some drugs of abuse. Phillips et al. (2003) found dopamine (DA) transients in the nucleus accumbens (NAc) that coincided with each operant lever press for a cocaine reward, and when the VTA was electrically stimulated to produce DA transients in the NAc, rats reliably pressed the lever for the reward. Dopamine neurons in the VTA display both low-frequency tonic activity as well as phasic high-frequency bursts. Tsai et al. (2009) used the cell-type specificity and temporal control of firing possible with ChR2 to test the role of these two activity patterns in mediating reward. They selectively expressed ChR2 in DA-VTA neurons and compared the effects of tonic 1-Hz stimulation with phasic $50-\mathrm{Hz}$ stimulation on DA transients in the NAc, and on behavior in a conditioned place-preference (CPP) task. CPP is used to measure preferences for environmental stimuli that have been associated with reward. The investigators showed increased DA released in the NAc upon $50-\mathrm{Hz}$, but not $1-\mathrm{HZ}$, stimulation of the VTA. Moreover, mice learned to prefer the chamber in which they received $50-\mathrm{Hz}$ stimulation, but not $1-\mathrm{Hz}$ stimulation. These results confirm a causal role for this DA network in reward-based behavior and directly demonstrate the importance of the high-frequency activity of these neurons.

The functional anatomy of the brain has generally been defined by either the physical lesion or pharmacological silencing of electrical activity in a specific region. Lesion studies in 
humans and animal models have shown a critical role for the hippocampus in the formation of explicit or declarative memory (Squire and Bayley 2007). These studies detect a time-limited role for the hippocampus in memory such that remote memories (from months to years old depending on species) no longer require the hippocampus. This suggests an anatomical alteration in the structure of memory over time such that the hippocampus is no longer recruited during the recall of remote memory. This temporal amnesia gradient has been seen in contextual fear conditioning, where the animal learns to fear a specific location (context) when conditioned with a footshock in that context (Kim and Fanselow 1992; Anagnostaras et al. 1999). However, this idea is not without controversy because other groups have failed to detect a temporal amnesia gradient, instead finding a requirement for the hippocampus in memory retrieval even at remote time points (Riedel et al. 1999; Sutherland et al. 2008). Goshen et al. (2011) revisited this issue using the photo-activatable suppressor of neural activity eNpHR3.1 (Gradinaru et al. 2010) to allow a more temporally precise assessment of the role of the hippocampus in remote memory retrieval. They expressed eNpHR3.1 in excitatory CA1 neurons using viral transfection and determined the effects of silencing this subregion on the retrieval of remote contextual fear memory. Consistent with previous studies, they found that when CA1 was selectively silenced during conditioning or during retrieval of a recent, 24-h memory, it impaired performance on the contextual version of the task. Inhibition of CA1 left intact auditory-cued fear memory, while silencing of the basolateral amygdala (BLA) prevented both contextual and cued fear conditioning, consistent with the known anatomy of these tasks. However, when the CA1 neurons were silenced $30 \mathrm{~d}$ following the initial learning, the animals also showed impaired memory retrieval. This result is at odds with many previous studies showing that contextual fear memory is insensitive to hippocampal lesion at this remote time point. One possibility is that compensatory changes in the circuit following silencing or removal of the hippocampus allowed memory retrieval in the previous studies. To test this idea, the researchers silenced CA1 for $\sim 30$ min before retrieval testing, to recapitulate the extended suppression of CA1 in lesion studies, and found no contextual memory deficit at the remote time point. Interestingly, when CA1 was intermittently inhibited during retrieval testing, mice showed a memory deficit that precisely followed light administration.

Previous studies (Frankland et al. 2004) have shown that expression of the neural activity-induced immediate-early genes cfos and zif268 is elevated in the hippocampus, but not anterior cingulate cortex (ACC), during a 24-h memory retrieval test. During remote memory testing, the labeling is reversed with elevated $c$ fos in the ACC and baseline levels in the hippocampus. These results led to the suggestion that following a 30-d incubation period, the memory is accessible using cortical circuits without involvement of the hippocampus. Goshen et al. (2011) showed that brief inhibition of CA1 immediately before remote retrieval testing, which results in memory impairment, led to a slight decrease of cfos expression in CA1 and a large decrease in ACC relative to controls. Prolonged, 30-min inhibition of CA1 before remote memory testing, which does not affect behavioral memory performance, again led to a reduction of $c$ fos expression in CA1, but in contrast to brief inhibition, resulted in highly elevated expression of $c$ fos in the ACC compared with control mice. These results show rapid (within $30 \mathrm{~min}$ ) adaptability in the circuit computations that are performed to produce the same cognitive outcome. These results provide a fundamentally different view of the role of the hippocampus in the intact brain and demonstrate the utility of rapid optogenetic manipulation in the functional dissection of neural circuits.

\section{Genetic manipulation of memory circuits}

In the preceding section, we discussed studies in which neurons were transfected using viral vectors that can be designed to target a specific cell type but label indiscriminately all cells within the target class. A common view of a neural circuit is that it is defined anatomically by the afferent, efferent, and local connections within a given structure. However, this may not capture the functional differences between apparently identical neurons that are the result of plasticity over the lifetime of the animal. As we have seen from studies in the hippocampus, motor cortex, and hypothalamus, excitatory neurons within a given region participate in encoding different information, and these specific neural representations are sparse and anatomically intermixed. A more functional view of a neural circuit is that it is defined by the environmental contingencies that stimulate electrical activity. In this section, we discuss two techniques that allow the targeting of genetic changes to sparse neural population based on their activity in response to specific environmental stimuli. This provides a means of altering anatomically dispersed circuits based on behaviorally relevant electrical response profiles.

While the hippocampus is required for contextual fear conditioning, the amygdala is critical for both contextual and cued conditioning. In fear conditioning to a discrete auditory tone, $\sim 70 \%$ of neurons in the lateral amygdala (LA) of freely behaving rats showed a greater response to the conditioned tone after it was paired with footshock. The increase in neural responsiveness immediately preceded or occurred simultaneously with changes in behavior (learned freezing response to the tone). Which neurons are altered with this form of learning, and how are they selected? Is the circuit hardwired such that specific subsets of neurons are always recruited for tone-shock associations, or is there flexibility in these circuits?

Han et al. (2007) used a clever approach to show that the amygdala neurons recruited with fear conditioning are not fixed, but arise through a competition between neurons. The investigators used viral transfection to deliver an activated form of the transcription factor CREB to $\sim 20 \%$ of neurons in the LA. Previous genetic studies had shown that CREB played an important role in fear conditioning and that viral transfection could rescue the learning deficits in CREB knockout mice. How could restoring CREB to such a small number of neurons rescue the learning deficit when $80 \%$ of neurons would still lack CREB? Han and coworkers hypothesized that the genetically rescued neurons might be preferentially recruited to encoding the fear memory. To test this idea, they used labeling with the neural activity-dependent gene arc to determine which neurons were activated with learning in the virally transfected mice. While CREB transfection did not alter the total level of arc expression following fear conditioning, arc activation was three times as likely to occur in CREB-overexpressing neurons than in neighboring uninfected neurons. This result was general and also occurred in wild-type mice and following transfection with either the wild-type or genetically activated forms of CREB. This suggests that neurons expressing the highest levels of CREB are selectively recruited into the fear memory trace.

Two studies went on to examine the mechanism of the circuit selection bias induced by CREB overexpression and to directly test the role of these neurons in the fear memory (Han et al. 2009; Zhou et al. 2009). CREB is a transcription factor, and it was unclear how it could directly affect the activity of neurons at the time of learning. Direct recording from the CREB-overexpressing neurons showed an increase in excitability. Thus, it is not CREB per se that is likely critical for the selective recruitment of these neurons with learning but their increased excitability relative to their neighbors. Do these more excitable cells selectively participate in the fear memory, or are they more readily labeled with arc due to their increased 
excitability? To examine the necessity of CREB-expressing neurons in memory recall, the viral vectors were constructed to deliver both CREB and an inducible silencer of the infected neurons. Han et al. (2009) overexpressed CREB along with the diphtheria toxin receptor (DTR) to allow the selective ablation of the infected neurons following diphtheria toxin administration. Zhou et al. (2009) used the same approach with expression of the allatostatin receptor (AlstR) to allow electrical silencing of the transfected neurons. Following fear conditioning, the CREB-overexpressing neurons were selectively labeled with arc showing their recruitment with learning. Silencing or ablation of these neurons impaired performance in a memory recall test. The effect was specific to the learned association and did not alter performance on memories encoded before the transfection. These results present a novel view of memory circuits, at least in auditory fear conditioning. In the LA, 70\% of neurons are responsive to auditory stimulation and thus hardwired to potentially participate in encoding the fear association. At the time of learning, there are intrinsic differences in excitability within this population of neurons, either based on CREB activity levels or other factors, and it is the subpopulation of more excitable neurons that is selectively recruited to encoding the memory. It will be interesting to determine whether this is a general mechanism used in other brain regions and behavioral tasks. Excitability-based selection of memory circuits has several implications for memory allocation. For example, in forming second-order associations, the recall of the first association would increase the excitation of neurons that participate in that memory and make them more likely to encode the new association, providing a mechanism for linking relevant information into a schema.

A critical unanswered question in neuroscience is how the brain represents complex percepts or memories. Viskontas et al. (2009) performed in vivo unit recordings in the anterior hippocampus and amygdala of human patients and found individual neurons that selectively responded to a picture, a written name, and a spoken name of a specific person. Not only were cells activated by well-known people that may have been stored as longterm memories in the patients, but also by multimodal presentations of the researchers performing the recordings, whom the patients had only known for a few days. This would seem to suggest that these neurons can encode the representation of a specific individual. But how can this be tested, and what are the parameters of neural activity that are necessary for recognition? If one could artificially fire this specific population of neurons, would the thought of that individual be brought to mind without specific sensory cues? How many neurons need to be fired to produce the percept, at what frequency, with what temporal coordination? Understanding these basic principles mediating complex representations is a critical step in understanding how the brain is organized to represent the world and how this is modified with experience.

This type of question can begin to be addressed using an approach to allow the genetic alteration of neurons based on their activity at a specific point in time. The neural activity-dependent genes cfos and arc have been used extensively to map environmentally induced brain activity in animal models. Reijmers et al. (2007) used the promoter for cfos in conjunction with the tetracycline regulatory system to generate mice in which the expression of any transgene could be driven into electrically active neurons. The approach uses two transgenes as shown in Figure $1 \mathrm{~A}$. In the first transgene the $c$ fos promoter is used to drive expression of the doxycycline (Dox)-regulated transcription factor (tTA) linking its expression to neural activity. The second transgene carries any tetO-promoter-linked gene. In double transgenic animals, the expression of tTA will be driven into active neurons via the cfos promoter, and this, in turn, will activate expression of the tetO-linked gene. The timing of the genetic tagging can
A

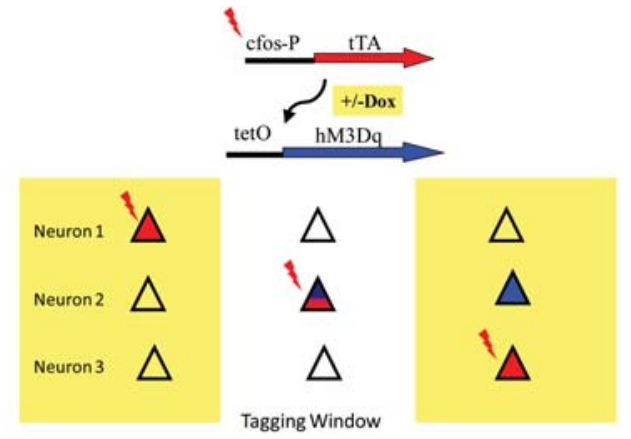

B
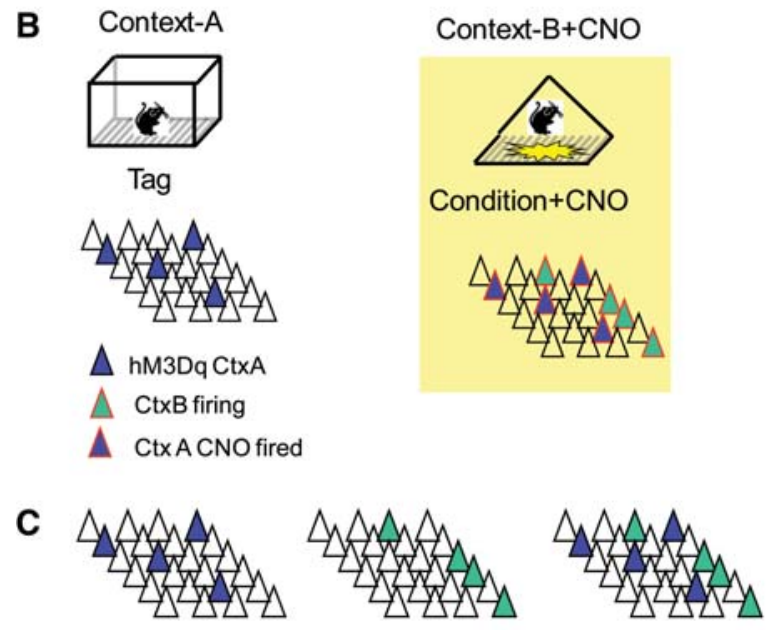

CNO-No Recall CtxB-No Recall CtxB+CNO-Recall

Figure 1. Generation of a hybrid memory representation. (A) The two transgenes required for activity-based genetic alteration. The first transgene links tTA to neural activity via the cfos promoter. The second transgene carries the hM3Dq DREADD receptor linked to the tTA-responsive promoter (tetO). In the absence of doxycycline (Dox, yellow) the tetOlinked $h M 3 D q$ gene is expressed in neurons that are sufficiently electrically active to drive cfos expression. tTA (red); hM3Dq (blue); electrically active neuron (bolt). (B) Conditioning protocol. Mice were exposed to context A (CtxA) to tag active neurons with $\mathrm{hM} 3 \mathrm{Dq}$ and fear-conditioned in context $\mathrm{B}(\mathrm{CtxB})$ following systemic administration of CNO to activate the CtxA representation. (Yellow) Dox treatment. (C) Putative neural activity patterns and behavioral results in a $24-\mathrm{h}$ memory test.

be controlled by Dox. In an initial study, Reijmers et al. (2007) used activity-dependent expression of a long-lasting marker protein to demonstrate that neurons that were activated with fear conditioning in the amygdala were reactivated during retrieval of the memory and that the degree of circuit reactivation was correlated with the strength of the memory. This approach provides a general method for introducing any genetic alteration specifically into neurons that are activated at a given point in time or by a specific sensory stimulus or behavioral contingency.

Garner et al. (2012) used cfos-based genetic tagging to drive expression of a $\mathrm{G}_{\mathrm{q}}$-protein-coupled muscarinic receptor, $\mathrm{hM}_{3} \mathrm{D}_{\mathrm{q}}$, engineered to be unresponsive to acetylcholine but activated by the biologically inert compound clozapine-N-oxide (CNO). When expressed in hippocampal pyramidal neurons, activation of the $\mathrm{hM}_{3} \mathrm{D}_{\mathrm{q}}$ receptor results in an $8-\mathrm{mV}$ depolarization and increased action potential firing (Armbruster et al. 2007; Alexander et al. 2009). The investigators used this approach to examine the circuits underlying the representation of a complex environment (Fig. 1). Mice were exposed to a novel environment (context A) with distinct visual, olfactory, and tactile cues, and 
the neurons activated were labeled with $\mathrm{hM}_{3} \mathrm{D}_{\mathrm{q}}$. The animals were then fear-conditioned in a separate environment (context B) following administration of CNO to concurrently fire the $\mathrm{hM}_{3} \mathrm{D}_{\mathrm{q}^{-}}$ tagged neurons representing context A. Animals did not form a fear memory for context A as might be expected if the CNO-based firing of those neurons was sufficient on its own to encode the neural representation of that context. The animals also did not form a memory for context B (the context in which they were shocked), showing that the firing of the additional context A neurons during conditioning interfered with normal memory encoding. What was surprising is that the mice formed a fear memory for context $\mathrm{B}$ that was only expressed when exposure to context $\mathrm{B}$ coincided with $\mathrm{CNO}$-induced neural activity from context A. Neither exposure to context B nor synthetic activation of context A neurons alone was sufficient to allow expression of the fear memory. This suggests that the animals formed a hybrid representation in which the activity of the context A neurons was incorporated as a necessary component of the memory.

The specificity of the hybrid representation was shown in two ways. First, the animals were conditioned to produce a hybrid representation incorporating context $\mathrm{A}$-labeled neurons, and the expression of $\mathrm{hM}_{3} \mathrm{D}_{\mathrm{q}}$ was allowed to turn over in those cells and $\mathrm{a}$ new ensemble of neurons was labeled by exposing the animals to a distinct context $\mathrm{C}$. Activation of context $\mathrm{C}$ neurons did not support memory recall, suggesting that the learning was specific to the pattern of cells activated in context $A$. In a second experiment, the mice were pre-exposed to context $B$ itself to induce $\mathrm{hM}_{3} \mathrm{D}_{\mathrm{q}}$ expression in context $\mathrm{B}$ neurons. The logic of this condition was that if the animals used the context $B$ neurons to represent the environment, then synthetic depolarization of those neurons during conditioning would not interfere with, and might even facilitate, memory encoding. Under these conditions, the animals developed a normal fear memory for context B even with CNO-based depolarization of the context $\mathrm{B}$ ensemble during conditioning.

What is the physiological relevance of these highly artificial manipulations of neural activity to normal information encoding in the brain? In the absence of specific sensory stimulation, the brain is not silent but displays significant spontaneous activity. In vivo recording studies suggest that this activity is not random but is skewed toward the coordinated reactivation of neural ensembles activated by previous experiences (Kenet et al. 2003; Karlsson and Frank 2009). In a sense, this internal spontaneous activity is a replay of previously learned information. In these experiments, a set of neurons activated by a previous experience (context A) is specifically reactivated during new learning and becomes incorporated into the new memory. The integration of internally generated patterns of activity with new learning through sensory experience could provide a mechanism for linking old and new information to build complex associations.

Another recent study also used the cfos-based genetic tagging approach to investigate contextual representations in fear conditioning (Liu et al. 2012). The investigators used the cfos-tTA transgenic mouse to control expression of a tetO-linked ChR2. In this case, the ChR2 was delivered specifically to neurons in the dentate gyrus (DG) using a viral vector. Animals were fear-conditioned, and the activated ensemble of neurons in the DG was genetically tagged with ChR2. This ensemble of neurons was then artificially activated with light while the animals were in a neutral context, and the mice developed a significant fear response. This suggests that the artificial stimulation of the pattern of DG neurons activated during initial learning was sufficient to produce recall of the contextual fear memory. This result suggests that at least a component of the context representation for fear memory is located in the DG.

The combination of genetically encoded regulators of neural activity with expression in neurons based on natural, environ- mentally evoked, activity patterns provides a means to begin to test the functional relevance of these natural ensembles in encoding complex representations. These initial two studies suggest a strong spatial coding mechanism with the pattern of stimulated neurons critical for recall. In neither case is the temporal pattern of neural firing or the relationship of action potential firing between neurons specifically controlled, suggesting that for these particular tasks, this type of higher-order information is not critical. The combination of new techniques for assessing neural activity patterns with high spatial resolution and manipulating the activity of specific neural circuits has opened up the possibility of directly testing the mechanisms by which the brain encodes and represents information.

\section{References}

Alexander GM, Rogan SC, Abbas AI, Armbruster BN, Pei Y, Allen JA, Nonneman RJ, Hartmann J, Moy SS, Nicolelis MA, et al. 2009. Remote control of neuronal activity in transgenic mice expressing evolved $\mathrm{G}$ protein-coupled receptors. Neuron 63: 27-39.

Anagnostaras SG, Maren S, Fanselow MS. 1999. Temporally graded retrograde amnesia of contextual fear after hippocampal damage in rats: Within-subjects examination. J Neurosci 19: 1106-1114.

Armbruster BN, Li X, Pausch MH, Herlitze S, Roth BL. 2007. Evolving the lock to fit the key to create a family of $G$ protein-coupled receptors potently activated by an inert ligand. Proc Natl Acad Sci 104: 5163-5168.

Boyden ES, Zhang F, Bamberg E, Nagel G, Deisseroth K. 2005. Millisecond-timescale, genetically targeted optical control of neural activity. Nat Neurosci 8: 1263-1268.

Buzsaki G. 2004. Large-scale recording of neuronal ensembles. Nat Neurosci 7: $446-451$.

Chow BY, Han X, Dobry AS, Qian X, Chuong AS, Li M, Henninger MA, Belfort GM, Lin Y, Monahan PE, et al. 2010. High-performance genetically targetable optical neural silencing by light-driven proton pumps. Nature 463: 98-102.

Dombeck DA, Harvey CD, Tian L, Looger LL, Tank DW. 2010. Functional imaging of hippocampal place cells at cellular resolution during virtual navigation. Nat Neurosci 13: 1433-1440.

Flusberg BA, Nimmerjahn A, Cocker ED, Mukamel EA, Barretto RP, Ko TH, Burns LD, Jung JC, Schnitzer MJ. 2008. High-speed, miniaturized fluorescence microscopy in freely moving mice. Nat Methods 5: 935-938.

Frankland PW, Bontempi B, Talton LE, Kaczmarek L, Silva AJ. 2004. The involvement of the anterior cingulate cortex in remote contextual fear memory. Science 304: 881-883.

Garner AR, Rowland DC, Hwang SY, Baumgaertel K, Roth BL, Kentros C, Mayford M. 2012. Generation of a synthetic memory trace. Science 335: 1513-1516.

Goshen I, Brodsky M, Prakash R, Wallace J, Gradinaru V, Ramakrishnan C, Deisseroth K. 2011. Dynamics of retrieval strategies for remote memories. Cell 147: 678-689.

Gradinaru V, Zhang F, Ramakrishnan C, Mattis J, Prakash R, Diester I, Goshen I, Thompson KR, Deisseroth K. 2010. Molecular and cellular approaches for diversifying and extending optogenetics. Cell 141: $154-165$.

Han JH, Kushner SA, Yiu AP, Cole CJ, Matynia A, Brown RA, Neve RL, Guzowski JF, Silva AJ, Josselyn SA. 2007. Neuronal competition and selection during memory formation. Science 316: 457-460.

Han JH, Kushner SA, Yiu AP, Hsiang HL, Buch T, Waisman A, Bontempi B, Neve RL, Frankland PW, Josselyn SA. 2009. Selective erasure of a fear memory. Science 323: 1492-1496.

Huber D, Petreanu L, Ghitani N, Ranade S, Hromadka T, Mainen Z, Svoboda K. 2008. Sparse optical microstimulation in barrel cortex drives learned behaviour in freely moving mice. Nature 451: 61-64.

Karlsson MP, Frank LM. 2009. Awake replay of remote experiences in the hippocampus. Nat Neurosci 12: 913-918.

Kenet T, Bibitchkov D, Tsodyks M, Grinvald A, Arieli A. 2003 Spontaneously emerging cortical representations of visual attributes. Nature 425: 954-956.

Kim JJ, Fanselow MS. 1992. Modality-specific retrograde amnesia of fear. Science 256: 675-677.

Komiyama T, Sato TR, O'Connor DH, Zhang YX, Huber D, Hooks BM, Gabitto M, Svoboda K. 2010. Learning-related fine-scale specificity imaged in motor cortex circuits of behaving mice. Nature 464: 1182-1186.

Lechner HA, Lein ES, Callaway EM. 2002. A genetic method for selective and quickly reversible silencing of mammalian neurons. J Neurosci 22: 5287-5290. 
Lerchner W, Xiao C, Nashmi R, Slimko EM, van Trigt L, Lester HA, Anderson DJ. 2007. Reversible silencing of neuronal excitability in behaving mice by a genetically targeted, ivermectin-gated $\mathrm{Cl}^{-}$channel. Neuron 54: 35-49.

Lin D, Boyle MP, Dollar P, Lee H, Lein ES, Perona P, Anderson DJ. 2011. Functional identification of an aggression locus in the mouse hypothalamus. Nature 470: 221-226.

Liu X, Ramirez S, Pang PT, Puryear CB, Govindarajan A, Deisseroth K, Tonegawa S. 2012. Optogenetic stimulation of a hippocampal engram activates fear memory recall. Nature 484: 381-385.

Luo L, Callaway EM, Svoboda K. 2008. Genetic dissection of neural circuits. Neuron 57: 634-660.

Phillips GD, Setzu E, Vugler A, Hitchcott PK. 2003. Immunohistochemical assessment of mesotelencephalic dopamine activity during the acquisition and expression of Pavlovian versus instrumental behaviours. Neuroscience 117: 755-767.

Reijmers LG, Perkins BL, Matsuo N, Mayford M. 2007. Localization of a stable neural correlate of associative memory. Science 317: 1230-1233.

Riedel G, Micheau J, Lam AG, Roloff EL, Martin SJ, Bridge H, de Hoz L, Poeschel B, McCulloch J, Morris RG. 1999. Reversible neural inactivation reveals hippocampal participation in several memory processes. Nat Neurosci 2: 898-905.

Slimko EM, McKinney S, Anderson DJ, Davidson N, Lester HA. 2002. Selective electrical silencing of mammalian neurons in vitro by the use of invertebrate ligand-gated chloride channels. J Neurosci 22: $7373-7379$.
Squire LR, Bayley PJ. 2007. The neuroscience of remote memory. Curr Opin Neurobiol 17: 185-196.

Sutherland RJ, O'Brien J, Lehmann H. 2008. Absence of systems consolidation of fear memories after dorsal, ventral, or complete hippocampal damage. Hippocampus 18: 710-718.

Tian L, Hires SA, Mao T, Huber D, Chiappe ME, Chalasani SH, Petreanu L, Akerboom J, McKinney SA, Schreiter ER, et al. 2009. Imaging neural activity in worms, flies and mice with improved GCaMP calcium indicators. Nat Methods 6: 875-881.

Tsai HC, Zhang F, Adamantidis A, Stuber GD, Bonci A, de Lecea L, Deisseroth K. 2009. Phasic firing in dopaminergic neurons is sufficient for behavioral conditioning. Science 324: 1080-1084.

Tye KM, Prakash R, Kim SY, Fenno LE, Grosenick L, Zarabi H, Thompson KR, Gradinaru V, Ramakrishnan C, Deisseroth K. 2011. Amygdala circuitry mediating reversible and bidirectional control of anxiety. Nature 471: 358-362.

Viskontas IV, Quiroga RQ, Fried I. 2009. Human medial temporal lobe neurons respond preferentially to personally relevant images. Proc Natl Acad Sci 106: 21329-21334.

Zhou Y, Won J, Karlsson MG, Zhou M, Rogerson T, Balaji J, Neve R, Poirazi P, Silva AJ. 2009. CREB regulates excitability and the allocation of memory to subsets of neurons in the amygdala. Nat Neurosci 12: $1438-1443$.

Received May 7, 2012; accepted in revised form June 1, 2012. 


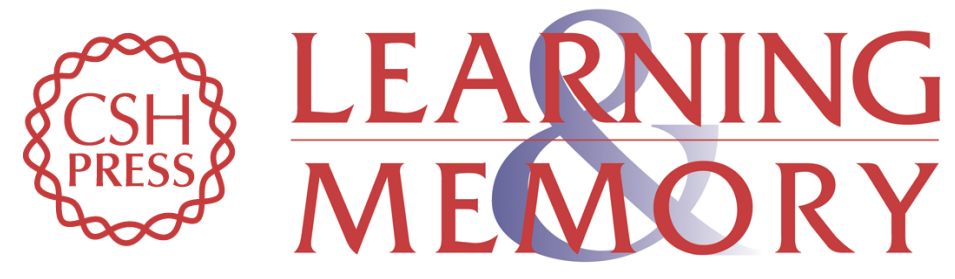

\section{New approaches to neural circuits in behavior}

Aleena Garner and Mark Mayford

Learn. Mem. 2012, 19:

Access the most recent version at doi:10.1101//m.025049.111

References This article cites 35 articles, 12 of which can be accessed free at: http://learnmem.cshlp.org/content/19/9/385.full.html\#ref-list-1

License

Email Alerting Receive free email alerts when new articles cite this article - sign up in the box at the Service top right corner of the article or click here. 